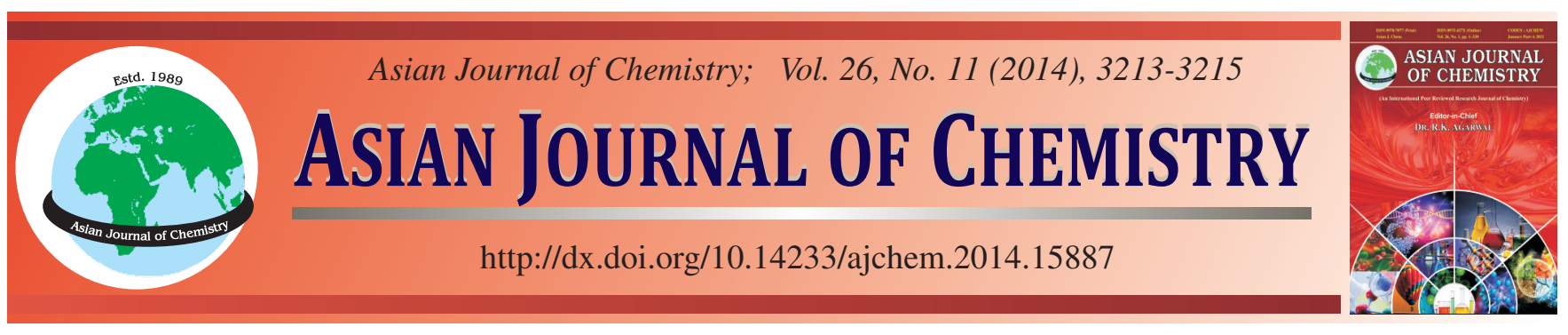

\title{
Preparation and Characterization of Synthetic Iron Oxide Coated Sand as Heterogeneous Fenton-Like Catalyst
}

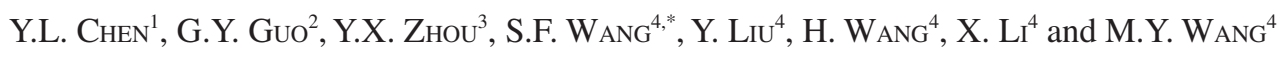

\begin{abstract}
${ }^{1}$ School of Chemistry and Chemical Engineering, Guangxi University, Nanning 530005, Guangxi Province, P.R. China ${ }^{2}$ Nanning Environmental Sanitation Administration Department, Guangxi, P.R. China

${ }^{3}$ Guangxi Bossco Environmental Protection Technology Co., Ltd., Nanning, Guangxi Province, P.R. China

${ }^{4}$ School of Light Industry and Food Engineering, Guangxi University, Nanning 530005, Guangxi Province, P.R. China
\end{abstract}

*Corresponding author: Fax: +86 771 3237097; Tel: +86 771 3237301; E-mail: wangsf@gxu.edu.cn

A new synthesis iron oxide coated sand catalyst (SCS) was synthesized by oil bath evaporation method. The physicochemical characteristics of the SCS were evaluated by various techniques such as SEM and ASAP. Particle size is about $100 \mathrm{~nm}$; specific surface area is $3.1347 \mathrm{~m}^{2} / \mathrm{g}$; surface iron oxide crystalline is hematite $\left(\alpha-\mathrm{Fe}_{2} \mathrm{O}_{3}\right)$. Synthetic iron oxide coated sand catalyst had well acidic and alkali resistances and the contents of Fe was determined by atomic spectrophotometer was $8.014 \mathrm{mg} \mathrm{Fe} / \mathrm{g}$.

Keywords: Erythromycin, Synthesis iron oxide coated sand, Heterogeneous Fenton.

\section{INTRODUCTION}

The heterogeneous Fenton-like catalyst was ties in catalytic activity and stability. All kinds of catalysts was divided into two parts: the iron powder and iron oxide ${ }^{1,2}$; and the iron-coated solid catalyst, such as $\mathrm{Al}_{2} \mathrm{O}_{3}{ }^{3}$, zeolite ${ }^{4}$, ion-exchange resin ${ }^{5}$, silica sand ${ }^{6}$, clay ${ }^{7}$, etc. The main iron oxide minerals were goethite $(\alpha-\mathrm{FeOOH})$, akaganeite $(\beta-\mathrm{FeOOH})$, lepidocrokite $(\gamma$-FeOOH $)$, hematite $\left(\alpha-\mathrm{Fe}_{2} \mathrm{O}_{3}\right)$ and the modifiers ${ }^{8-10}$.

The types of carriers of synthesis supported iron oxide catalyst (SCS) mainly include natural solid inorganic material, macromolecule organic materials and membranes. The common supports of the natural solid inorganic material were zeolite, $\operatorname{resin}^{11}$, activated carbon ${ }^{12,13}$, silica $^{14,15}$, carbon fiber $^{16}$, etc. The natural solid inorganic material had large specific surface area and good anti-oxidation ability. Otherwise, the cation resin of macromolecule organic materials often serves to organic supporters. In 1998, the iron-coated Nafion membrane was studied on printing and dyeing wastewater treatment. The results indicated that the iron-coated Nafion membrane was easily recycled and high oxidative ability. But the much higher cost had restricted its industrialization applications ${ }^{17,18}$.

The physical and chemical influence factors in iron-coated catalysts, which determined the types of synthesis supported iron oxide catalyst, were called supported parameters, such as $\mathrm{pH}$, temperature, concentration of ferric salts and time in supported periods. Otherwise, the removal of cationic and anti- acid alkali performance of SCS was effected by supported parameters. Lo et al. ${ }^{19}$ proposed that the $\mathrm{pH}$ and temperature were the most important to effect the types of SCS and the removal rate. Along with the increase of $\mathrm{pH}$, the anti-acid alkali performance was worse and worse, the dissolution decreased to $25 \%$; when temperature increase, the anti-acid alkali performance increased and so did removal rate. At low oxygen or hypoxia of aquatic and terrestrial environment, the surface activity of SCS showed better ${ }^{20}$ and the specific surface area and surface charge was higher. Therefore, SCS was available for water treatment ${ }^{21}$.

\section{EXPERIMENTAL}

The method for the synthesis of iron coated sand (SCS) is to add the sand into ferric salt solution, evaporating with oil bath.

Carrier selection: Granular carrier was the most important way of making SCS in various kinds of iron oxide carriers. Because the Fenton system has strong oxidation, the carriers with stable performance and powerful antioxidants must be chosen. Silica, activated carbon, biological ceramic and zeolite were chosen as catalyst supports. Using the COD value of reaction process as the detection target, the treatment effect of four catalyzers were observed and compared.

Coated method: Three different coated methods of SCS, directed evaporation, alkaline deposition and oil bath evaporation were compared. 
Characterization of SCS: The physicochemical characteristics of the SCS were evaluated by SEM and ASAP.

\section{RESULTS AND DISCUSSION}

Carrier selection: The zeolite was pulverized easily during preparation. Therefore, the catalytic activity of three other catalysts was detected and analyzed.

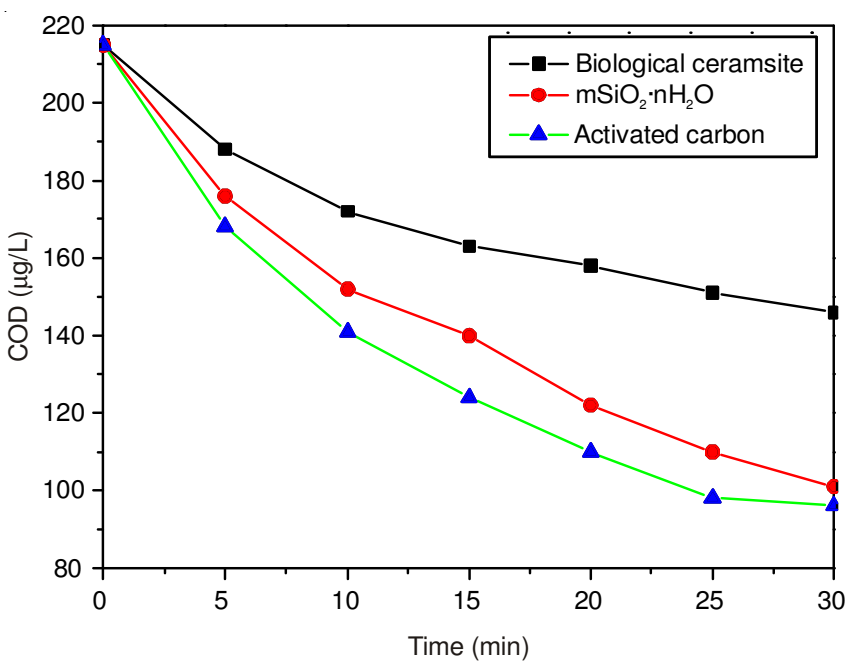

Fig.1. Effect of different carriers on catalytic activity of catalyst

The effects of carriers of sand and activated carbon on catalytic activity of catalyst were better than biological ceramist as shown in Fig. 1. However, the activated carbon catalyst easily dissolved. The iron-coated sand was chosen.

Coated method: Table-1 showed the degradation properties of SCS, the SCS coated using $\mathrm{Fe}\left(\mathrm{NO}_{3}\right)_{3}$ solution had better degradation than $\mathrm{FeCl}_{3}$ solution; the coating by alkaline evaporation method with lower iron value, due to the bad adhesion of iron oxide; while coated by directed evaporation was moisture and caking, the degradation effect was poor.

\section{TABLE-1}

COMPARISON OF DEGRADATION PROPERTIES OF SCS

$\begin{array}{lllllllll}\text { Sample No. } & \mathbf{1} & \mathbf{2} & \mathbf{3} & \mathbf{4} & \mathbf{5} & \mathbf{6} & \mathbf{7} & \mathbf{8}\end{array}$

$\begin{array}{lllllllll}\mathrm{COD}(\mathrm{mg} / \mathrm{L}) & 473 & 191 & 170 & 265 & 126 & 168 & 212 & 96\end{array}$

1-raw water; 2-Fenton reagent; 3-SCS using $\mathrm{Fe}\left(\mathrm{NO}_{3}\right)_{3}$ solution by directed evaporation; 4-SCS using $\mathrm{Fe}\left(\mathrm{NO}_{3}\right)_{3}$ solution by alkaline evaporation method; 5-SCS using $\mathrm{Fe}\left(\mathrm{NO}_{3}\right)_{3}$ solution by oil bath evaporation; 6-SCS using $\mathrm{FeCl}_{3}$ solution by directed evaporation; 7SCS using $\mathrm{FeCl}_{3}$ solution by alkaline evaporation method; 8-SCS using $\mathrm{FeCl}_{3}$ solution by oil bath evaporation

Characterization of SCS: The physicochemical characteristics of the SCS were evaluated by SEM and ASAP.

SEM: SEM images of the sand and SCS showed in Fig. 2. The components and the skeletal structure determined the surface configuration of SCS. The surface configuration of sand and SCS were investigated with SEM, which used to determine the type and particle size of iron oxide of the surface of SCS. Fig. 2 (1) showed the surface of sand by pickling was smooth, without any impurities. The most common surface configuration of sand was subangular, followed by sharp edges and shape, sub-rounded. It was identical with recorded in literatures ${ }^{22}$. Meantime, the concave and convex groove of sand not merely offered more load surface, but also increased the adhesive strength between iron oxide and sand. Fig. 2 (2) showed that the surface of SCS was rough. Because of the uniform iron oxide film was packed the sand. Hence, more active sites of SCS had the better degradation effect in Fenton system.

The surface of SCS magnified to 10000 times its ordinary size by SEM was showed in Fig. 2 (3). Iron oxide grain, with particle size $10^{2} \mathrm{~nm}$, evenly coated the surface of SCS. SCS become more reactive, because of mass of particles with larger porosity, which obtained large surface area and much more atoms exposed. On the one side, the adsorptivity of heavy metal, nitrogen and phosphorus could be increased. On the other hand, more unpaired electrons with high reactivity exposed, which reacted with other atoms and molecules easily ${ }^{23}$.

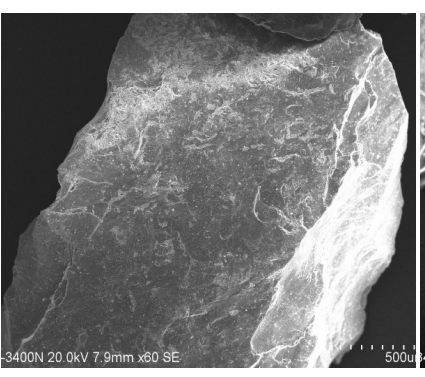

(1) Silica sand $(\times 60)$

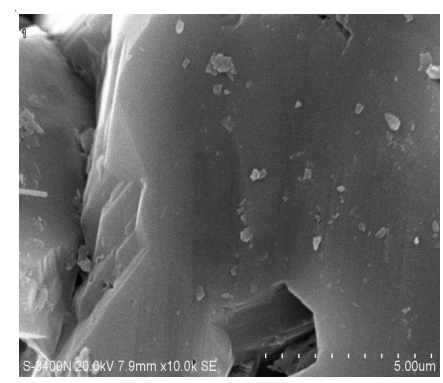

(3) Silica sand $(\times 10000)$

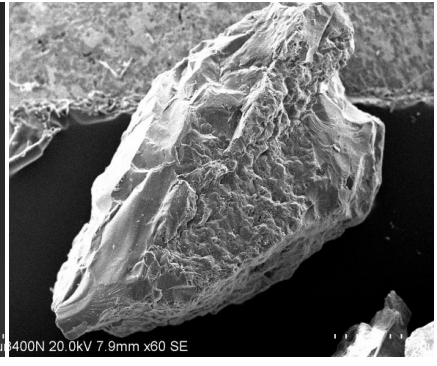

(2) $\operatorname{SCS}(\times 60)$

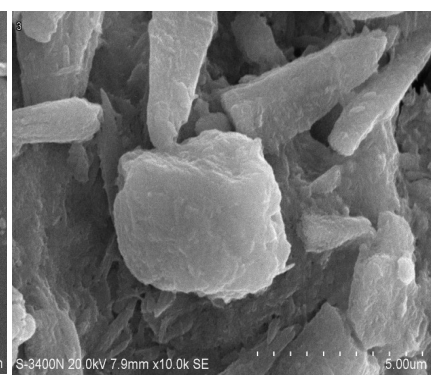

(4) $\operatorname{SCS}(\times 10000)$
Fig. 2. SEM images of the sand and SCS

ASAP: The main functions of SCS as a heterogeneous Fenton-like catalyst were absorption and oxidative degradation. Research showed that larger surface more adsorption sites and better absorption efficiency. The pore diameter and the pore-size distribution also had obviously effect on absorption efficiency. The macromolecule organic matters were difficult to degradation which was usually caused by the reason of too small pore diameter. On the contrary, too big pore diameter caused surface areas decline and the adsorption effect reduced. The ASAP method was used to determine the specific surface area, surface pore size and pore volume.

As the BET method described, Fig. 3 showed the curve fitting with $\frac{\mathrm{P}}{\mathrm{V}\left(\mathrm{P}_{\mathrm{o}}-\mathrm{P}\right)} v s . \frac{\mathrm{P}}{\mathrm{P}_{\mathrm{o}}}$ The slope and intercept of this linear fitting equation were $0.6228 \mathrm{~m}^{2} / \mathrm{g}, 3.1347 \mathrm{~m}^{2} / \mathrm{g}$, respectively. In conclusion, the surface area of SCS was about five times bigger than silica sand. The surface area of SCS increased significantly would enhance the adsorption. Compared with Fenton reagent, the solid-liquid separation of SCS 


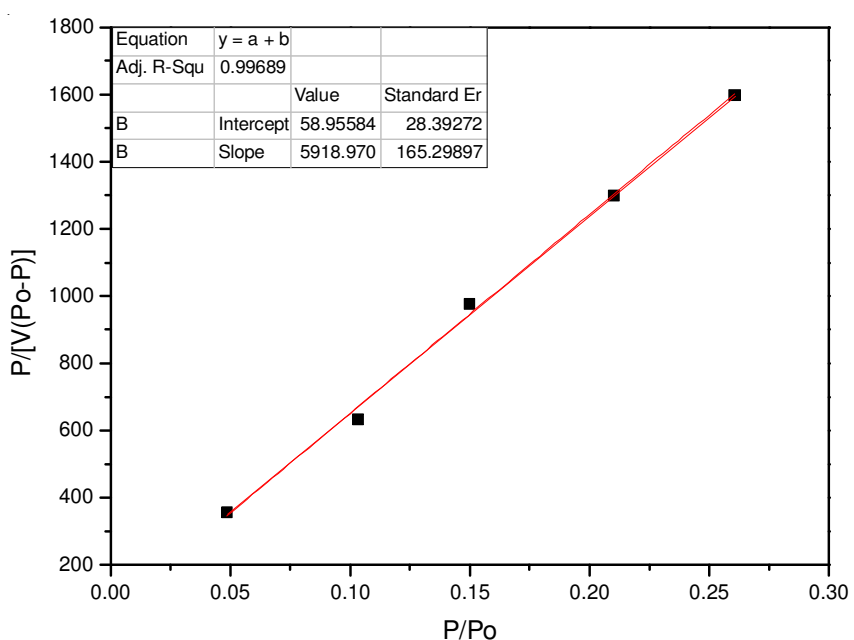

(1) Silica sand

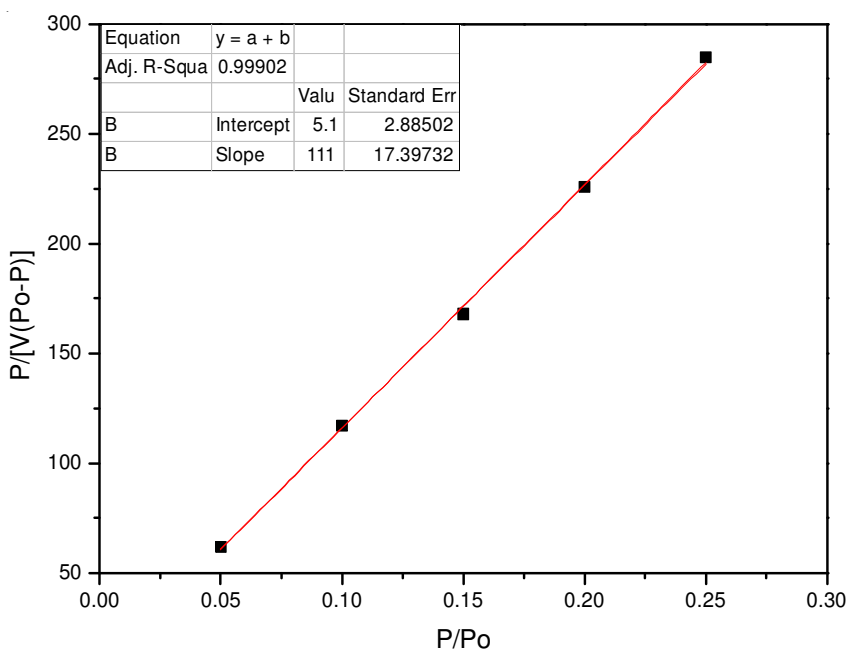

(2) SCS

Fig. 3. BET of silica sand and SCS

was much easier and the shortages of iron mud, such as large amount and refractory, had been solved.

\section{Conclusion}

Synthetic iron oxide coated sand catalyst was synthesized by evaporating ferric salt solution with oil bath and was characterized by SEM and ASAP. It showed that the particle size and the surface area were about $100 \mathrm{~nm}$ and $3.1347 \mathrm{~m}^{2} / \mathrm{g}$, respec- tively. Synthetic iron oxide coated sand catalyst was much stronger adsorption and more reactive. Compared with Fenton reagent, the solid-liquid separation of SCS was much easier and the amount of iron mud was depleted.

\section{ACKNOWLEDGEMENTS}

This work was supported by Guangxi University Projects, China (contract No.1201043).

\section{REFERENCES}

1. M.C. Lu, J.N. Chen and H.H. Huang, Chemosphere, 46, 131 (2002).

2. R. Andreozzi, A. D'Apuzzo and R. Marotta, Water Res., 36, 4691 (2002).

3. N. Al-Hayek and M. Doré, Water Res., 24, 973 (1990).

4. R. Gonzalez-Olmos, M.J. Martin, A. Georgi, F.-D. Kopinke, I. Oller and S. Malato, Appl. Catal. B, 125, 51 (2012).

5. R.-M. Liou, S.-H. Chen, M.-Y. Hung and C.-S. Hsu, Chemosphere, 55, 1271 (2004).

6 M.M. Benjamin, R.S. Sletten, R.P. Bailey and T. Bennett, Water Res., 30, 2609 (1996).

7. J. Barrault, C. Bouchoule, J.-M. Tatibouët, M. Abdellaoui, A. Majesté, I. Louloudi, N. Papayannakos and N.H. Gangas, Stud. Surf. Sci. Catal., 130, 794 (2000).

8. S. Lee, J. Oh and Y. Park, Bull. Korean Chem. Soc., 27, 489 (2006).

9. L.C.A. Oliveira, M. Goncalves, M.C. Guerreiro, T.C. Ramalho, J.D. Fabris, M.C. Pereira and K. Sapag, Appl. Catal. A, 316, 117 (2007).

10. I. Guimarães, L.C.A. Oliveira, P.F. Queiroz, T.C. Ramalho, M.C. Pereira, J.D. Fabris and J.D. Ardisson, Appl. Catal. A, 347, 89 (2008).

11. J.H. Ramirez, C.A. Costa, L.M. Madeira, G. Mata, M.A. Vicente, M.L. Rojas-Cervantes, A.J. López-Peinado and R.M. Martín-Aranda, Appl. Catal. B, 71, 44 (2007).

12. M.A. Fontecha-Cámara, M.A. Álvarez-Merino, F. Carrasco-Marín, M.V. López-Ramón and C. Moreno-Castilla, Appl. Catal. B, 101, 425 (2011).

13. T.D. Nguyen, N.H. Phan, M.H. Do and K.T. Ngo, J. Hazard. Mater., 185, 653 (2011).

14. S. Navalon, M. Alvaro and H. Garcia, Appl. Catal. B, 99, 1 (2010).

15. F. Martinez, G. Calleja, J.A. Melero and R. Molina, Appl. Catal. B, 70, 452 (2007)

16. Q. Liao, J. Sun and L. Gao, Colloids Surf. A, 345, 95 (2009).

17. Y. Zhang, C. He, V.K. Sharma, X.- Li, S. Tian and Y. Xiong, Sep. Purif. Technol., 80, 45 (2011).

18. M. Remy, V. Potier, H. Temmink and W. Rulkens, Water Res., 44, 861 (2010).

19. S.H. Lin and C.C. Lo, Water Res., 31, 2050 (1997).

20. G. Sposito, The Surface Chemistry of Soils, Oxford University Press, New York (1984).

21. K. Hanna, T. Kone and G. Medjahdi, Catal. Commun., 9, 955 (2008).

22. Z. Li, L.Q. Li, Y.N. Zeng et al., Study of loess in Xi Ning. Qing Hai Geology. 1996:1-10.

23. Z. Shi, G.M. Xu and J. Deng, Water Environ. Res., 1, 639 (2009). 